\title{
BMJ Open Risk communication with Arab patients with cancer: a qualitative study of nurses and pharmacists
}

\author{
Kerry Wilbur, ${ }^{1}$ Alya Babiker, ${ }^{1}$ Maha Al-Okka, ${ }^{1}$ Ebaa Jumaat, ${ }^{1}$ \\ Sumaya M Al Saadi Al-Yafei, ${ }^{2}$ Abdulqadir J Nashwan ${ }^{3}$
}

To cite: Wilbur K, Babiker A Al-Okka M, et al. Risk communication with Arab patients with cancer: a qualitative study of nurses and pharmacists. BMJ Open 2015;5:e006890

doi:10.1136/bmjopen-2014006890

- Prepublication history and additional material is available. To view please visit the journal (http://dx.doi.org/ 10.1136/bmjopen-2014006890).

Received 17 October 2014 Revised 2 March 2015 Accepted 3 March 2015

\footnotetext{
${ }^{1}$ College of Pharmacy, Qatar University, Doha, Qatar ${ }^{2}$ Department of Pharmacy, National Center for Cancer Care and Research, Doha, Qatar

${ }^{3}$ Nurse Urgent Care Unit, National Center for Cancer Care and Research, Doha, Qatar
}

Correspondence to Dr Kerry Wilbur; kwilbur@qu.edu.qa

\section{ABSTRACT}

Objectives: To explore pharmacist and nurse views and experiences in educating patients regarding their treatment safety and tolerability as well as the roles of other professions in this regard.

Design: In this qualitative study, six focus group discussions were conducted.

Setting: The National Center for Cancer Care and Research in Qatar.

Participants: Eleven pharmacists and 22 nurses providing direct patient care.

Results: Concepts related to three key themes were drawn from the seeding questions and included factors for determining the level of risk they communicated: the specific treatment regimen in question; the patient; and their assessment of the patient. Patient-related considerations arose from additional subthemes; both nurses and pharmacists described aspects related to the perceived psychological health status of the patient, as well as anticipated comprehension, as ascertained by demonstrated education and language abilities. In all discussions, it was noted that physician and family non-disclosure of cancer diagnosis to the patient profoundly influenced the nature of information they provided. While a high level of cohesion in safety communication prioritisation among these two health disciplines was found, a number of pharmacists asserted a more formal role compared to informal and repeated teaching by nurses.

Conclusions: Nurses and pharmacists in this Middle East healthcare environment were not reluctant to discuss treatment side effects with patients and draw on similar professional judgements in prioritising treatment risk information. We found that they did not always recognise each other's informal educational encounters and that there are opportunities to explore increased collaboration in this regard to enhance the patient care experience.

\section{BACKGROUND}

Cancer care is increasingly complex and must draw on the distinct yet complementary skills of various health disciplines. Patients must not only contend with the realities of the

\section{Strengths and limitations of this study}

- Information thresholds for treatment risk communication with patients with cancer can vary across cultures, but little is known about practices in the Middle East.

- We used qualitative methodology to explore nurse and pharmacist perspectives on patient education regarding side effects and intolerances of cancer therapy.

- Although we do not measure observational data to support the self-reports, both health professional groups identified the need and means to frame risks appropriately and expressed beliefs in the patient's right to such information.

diagnosis and possible unfavourable prognosis, but also digest information related to complex and often debilitating treatments. ${ }^{1} 2$ The support of a multidisciplinary team then can be integral to addressing patient concerns and facilitating information exchange. In cancer care specifically, coordinated contribution by diverse health professionals increases delivery of evidence-based treatment and improves patient satisfaction. ${ }^{3-5}$

Results from countless studies have highlighted the value of providing adequate information to patients with cancer, which includes reports of decreased depression and anxiety; the promotion of self-care and adherence; as well as increased patient satisfaction with overall management. ${ }^{5-10}$ In particular, clinical teaching regarding the side effects of treatments and recommended management strategies is information consistently ranked high in importance by patients with cancer. ${ }^{11} 12$ Mental well-being was inversely related to the number of unmet information needs related to side effects and symptoms among long-term survivors of cancer. ${ }^{10}$ Patients reflecting at the conclusion of cancer treatment opined that frank information related to medication intolerances would have decreased their fear associated with chemotherapy. ${ }^{13}$ However, the 
desired level of such detail is different across cultures and very little of this work has been conducted among Arab patients with cancer. ${ }^{1415}$

The Middle East region is currently undergoing an expansion of specialised oncology treatment centres to address the rising incidence of cancer cases among their populations. ${ }^{16}$ The coordination of care among healthcare providers is experiencing a parallel evolution in these settings. Implementation of effective and integrated multidisciplinary team-based care for patients with cancer varies throughout the Middle Eastern region. ${ }^{17}$ Both pharmacists and nurses counsel patients on their medications in a variety of settings during encounters with patients with cancer in Qatar. They share responsibility to educate patients with cancer about the safety of their medications, but may be reluctant to offer detailed information regarding treatment side effects for fear of provoking nonadherence. A prior study among oncology nurses and physicians has demonstrated that health professionals may underestimate the information needs of patients with cancer. ${ }^{1} 1318$ Elsewhere, patients with cancer express a strong desire for information, but how is this addressed by providers in a Middle Eastern context? We sought to evaluate how pharmacists and nurses counsel patients regarding the potential side effects of their therapy and identify healthcare provider roles in this regard.

\section{METHODS}

A qualitative descriptive study design using focus group discussion for data collection was employed and chosen as an efficient and feasible means to record participant views. Such dialogue permits extensive responses about people's knowledge, attitudes and views and often precedes and informs the development of other qualitative means of seeking to determine individual opinions (surveys, questionnaires). ${ }^{19}$

Pharmacists $(\mathrm{n}=32)$ and nurses $(\mathrm{n}=263)$ providing care at the National Center for Cancer Care and Research (NCCCR) in Qatar were invited by email to participate.

The research purpose and methodology were reviewed verbally and in writing (consent form) with participants attending the interviews at NCCCR. A topic guide was developed following a comprehensive literature review of reports of other qualitative and quantitative assessments of education of patients with cancer pertaining to chemotherapy (see online supplementary appendix 1 ). The devised framework sought to explore consenting participant views and experiences in educating patients regarding the safety and tolerability of their treatment and the roles of multidisciplinary members in this regard. One author facilitated each interview with a second in attendance serving as a field note-taker. At the end of each discussion, participants were given the opportunity to ask additional questions or make further contributions. Following this closing, the facilitator and note-taker conducted a debriefing exercise, whereby the researchers confirmed the seating arrangement and associated coding of the participants, reflected on participant responses and finally any suggestions for improving subsequent data collection events. ${ }^{19}{ }^{20}$ Each audio taped focus group interview was conducted and transcribed by one of the investigators and transcriptions subsequently verified and finalised independently by a separate author.

The qualitative analysis of interviews was supported by NVivo10 (QSR International 2013) software. Transcripts were read through several times by all primary investigators to obtain the sense of the whole and then subjected to latent content analysis. Using thematic content analysis, the text was divided into words, sentences or paragraphs, related to each other through their content and context as units of meaning. ${ }^{19-21}$ The data were then coded and organised around interpreted subthemes and themes and based on comparisons of their similarities and differences. Working theories were consolidated by the primary investigators based on relevant topic characteristics associated with the means by which they prioritise and choose adverse effect information to communicate to patients. ${ }^{22}$

\section{FINDINGS}

Six separate focus group discussions lasting approximately 30-45 min were conducted-three each for pharmacists $(n=12)$ and nurses $(n=22$; table 1$)$.

Participants delivered patient care in inpatient and outpatient settings at NCCCR. The mean duration of experience in oncology care was 6.2 (SD 2.3) years for pharmacists and 9.9 years (SD 3.9) for nurses.

Participants were first asked to name the important adverse effects they would generally communicate to patients in their care. All groups consistently chose specific intolerances most prevalent with chemotherapy regimens: nausea, vomiting, diarrhoea; symptoms associated with immunosuppression (fever, chills); hair loss; and infertility. Nurses tended to emphasize infusionrelated reactions, such as flushing, hypotension and breathing difficulties. Both cohorts stressed that they did not share such side effects in isolation, but together with reassurances of their management.

We will advise them, for example, most of these side effects are reversible, and we have a lot of techniques to

\begin{tabular}{|c|c|c|}
\hline Discipline & Participants, n & $\begin{array}{l}\text { Years in oncology } \\
\text { practice (mean, range) }\end{array}$ \\
\hline \multicolumn{3}{|c|}{ Pharmacists } \\
\hline FG 1 & 3 & $6(1-7)$ \\
\hline FG 2 & 4 & $5(3-6)$ \\
\hline FG 3 & 5 & $7.5(5-11)$ \\
\hline \multicolumn{3}{|l|}{ Nurses } \\
\hline $\mathrm{FG} 1$ & 8 & $10.25(6-18)$ \\
\hline FG 2 & 8 & $9.5(2-18)$ \\
\hline FG 3 & 6 & $10(2-20)$ \\
\hline
\end{tabular}


control, we have medication for nausea and vomiting.

(Focus Group 1, Nurse 2)

When prompted next to offer how they would prioritise this communication, concepts and connections to three main themes were identified, describing factors related to: the specific regimen in question; the patient; and their assessment of the patient. Patient-related considerations were then further categorised into subthemes of health status, psychological status and education (figure 1).

\section{SPECIFIC REGIMEN}

Nurses and pharmacists both indicate giving patients information about the most common side effects that was further augmented according to the specific drugs in the particular protocol prescribed.

This [education] is starting from the protocol-we have ABVD protocol, most of the patients, like $70-80 \%$, have severe vomiting during this chemo. (Focus Group 3, Nurse 1)

The timing and location of drug administration also influenced this exchange. Patients admitted to NCCCR to receive the first cycle of parenteral chemotherapy routinely meet a member of the clinical pharmacy team and nurse educator for counselling. Most of the bedside nurses we interviewed identified only reinforcement of precautionary measures then at this point of care.

We are teaching the patient immediately if he has any fever or dizziness or anything, immediately to let us know, to go for medical attention. (Focus Group 1, Nurse 4)

Participants in all discussions elaborated that the information they disclosed was influenced by the sequence of the encounter with the patient during the course of their care.
Mostly I would start with the most common side effects and things that they can see within the first week of treatment and what are the things that they take precautions with, so they will not have an impact on their life within the initial cycles of chemotherapy. (Focus Group 3, Pharmacist 1)

It's a continuous process starting from first time to the last cycle... some [patients], they will get used to the information from the past experience, the past cycle [and] some will not. Some will be continuously asking and we have to continuously answer. (Focus Group 2, Nurse 3)

\section{PATIENT FACTORS}

\section{Health status}

A great deal of consideration for patient factors further directed the content of these health professionals' patient education. Determining if the prescribed regimen was curative or not, and if the patient was actually aware of his or her prognosis, were aspects very carefully weighed prior to patient interactions.

If a patient is taking doxorubicin, for example, and it is very well known that it causes cardiomyopathy [that may appear after two years] and the patient has life expectancy up to six months, I will not worry him more, not bother him with that side effect. (Focus Group 2, Pharmacist 5)

If the patient doesn't know the diagnosis, you can't start talking about chemotherapy; however, we give them information about side effects or how to manage it, but not in a detailed way. (Focus Group 2, Pharmacist 2)

Most participants agreed that the counselling they offered to patients was also in deference to the family's wishes and often involved non-disclosure of the medication's indication.

Sometimes we have problems with chemotherapy. Some patients, the relative doesn't want to tell the patient. We have one case actually-don't tell the patient that this is chemotherapy. (Focus Group 2, Nurse 7)

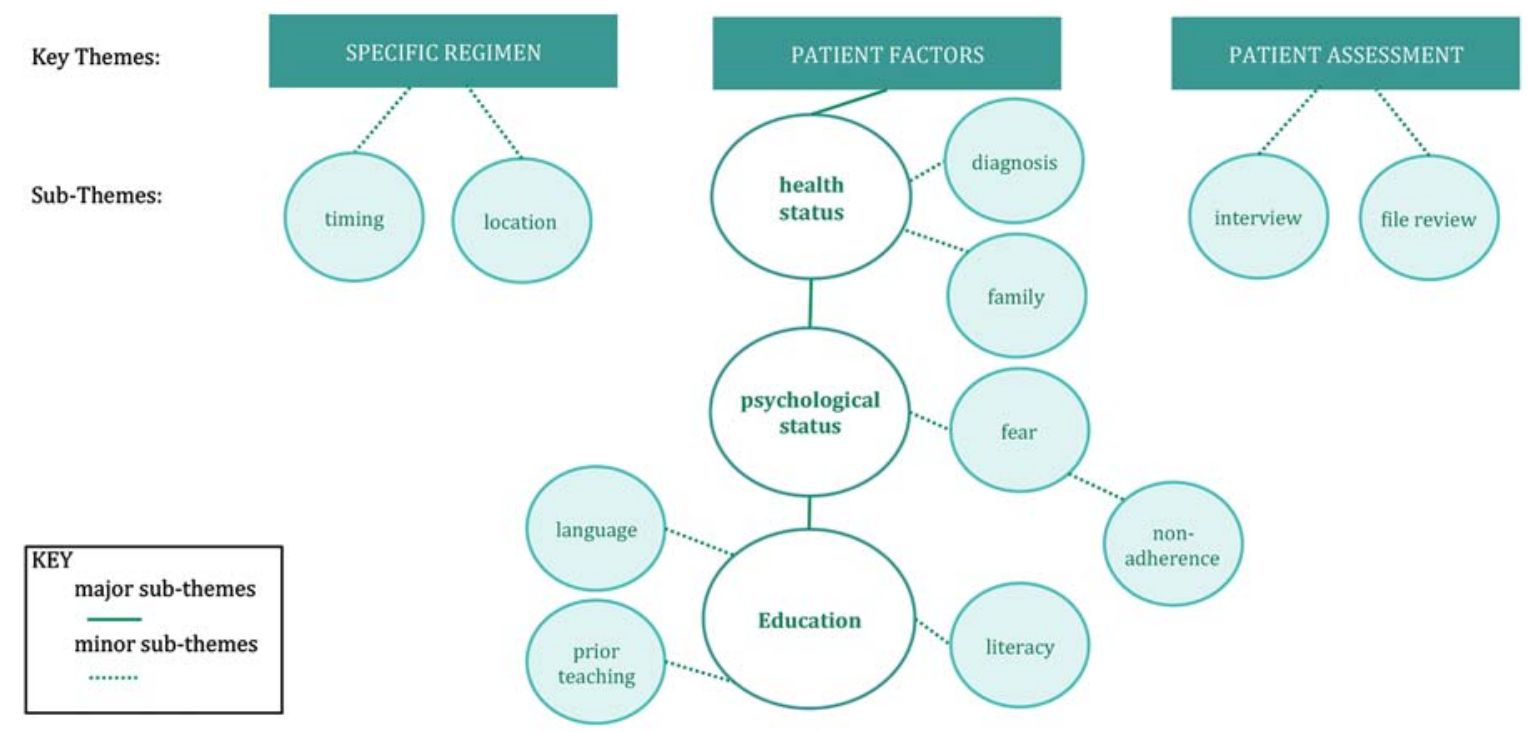

Figure 1 Conceptual diagram. Themes identified from focus group discussions. 


\section{Psychological status}

Both nurses and pharmacists described assuming a reassuring role in the care of their patients. Recognising the vulnerability of many patients with cancer receiving treatment, they tailored their education accordingly.

You know our patients, they are afraid of the chemotherapy, especially if we gave all the side effects at one time. At first, give at least the common things. (Focus Group 3, Nurse 2)

Sometimes they [patients] say they are afraid and 'I don't want to take the chemo', so we should choose which side effects and what information we should give. (Focus Group 1, Pharmacist 1)

While a number of participants acknowledged concerns that learning the risks associated therapy may dissuade patients from accepting treatment, a strong sense of responsibility to prepare their patients for the inevitable adverse effects emerged.

For the young also we will prepare them if they have long hair to start to cut, how to manage, because also body image it's very important at this age adolescence, teenagers or 13 even 20. If they are planning to have a baby for young ladies for example, for surgery, for mastectomy, we have to prepare them, we will not ignore this. (Focus Group 1, Pharmacist 2)

The point is like this, if we explain, some patients they are afraid, some patients they will not agree, but if you don't explain hair loss will be there, vomiting will be there, nausea will be there... if you don't explain it, once it occurs they will question the doctor or the nurse, 'why you didn't you tell us when we started?'. That's the problem. (Focus Group 2, Nurse 5)

\section{Education}

The versatility required for patient counselling was underlined by several descriptions of communication barriers with the diverse patient population in this setting, including literacy and mismatch between the care provider and the patient's native language.

When you are explaining to the patient, you must know the level of understanding; you will use the simplest language you can. (Focus Group 2, Nurse 6)

Some patients are not well educated at all some patients cannot read and write, so it's difficult to explain about neutropenia even about immunity in Arabic or English or whatever language, so sometimes it's very difficult to educate. (Focus Group 1, Pharmacist 2)

Pharmacists further underlined the importance of acknowledging prior teaching by other healthcare providers and the ability to adapt education to the expressed needs of the patient.

We tell them that we know that you have received education before but we need to know the extent of the knowledge to make sure that you understand fully all the aspects of the treatment side effects and to understand where are the areas that we need to focus on with you. (Focus Group 1, Pharmacist 3)

In the outpatient setting when you interview the patient he might come up with side effect that is his big concern. (Focus Group 2, Pharmacist 2)

\section{Patient assessment}

Determining the health and psychological status and education level of patients with cancer is accomplished through the professional judgement of participants. All stressed the unique nature of each care provider-patient therapeutic relationship and both nurses and pharmacists illustrated the straightforward yet effective interview approach to ascertain the aforementioned elements described.

Once you talk to them, you will be able to know whether they are expecting very little information or the medium or the vast. (Focus Group 2, Nurse 3)

Just ask the patient. We have a small conversation with the patient before starting to mention anything about the medication. We just have like a background conversation about the patient-what he knows from his physician, what he knows about his medication-according to this we will build what we have to say. (Focus Group 2, Pharmacist 3)

I would say also that two-way communication will also facilitate the issue. I usually go to the patient and ask the first question, 'do you know why you are here today?' (Focus Group 2, Pharmacist 4)

\section{EDUCATION ROLES OF HEALTHCARE PROVIDERS}

When participants were asked about their perceptions regarding the roles of different health professionals in explaining the adverse effects of cancer therapy, many identified how the introduction is usually by the physician who takes the patient consent for chemotherapy. Nurses posited that while the physician should assume the primary role in patient education, they would emphasise important adverse effects and extend other advice over the course of treatment, most notably patient reassurance.

The first time the doctor will [give information]-they are not convinced, they still have the doubts. But we are the front line, we will 'chill' them step-by-step. (Focus Group 1, Nurse 3)

Pharmacists corroborated the nurses' impression about patients' need for reinforcement following the physician visits and a number described receiving physician referral for such patient counselling. Some acknowledged the value of nurses as the front-line caregiver in evaluating patient drug tolerability, but most participants adhered firmly to their scope of practice as the optimal medication educators. 
For example, it's a weekend and there is no pharmacist available in the word and the patient will be discharged —she [nurse] just will sit the basic side effects. (Focus Group 1, Pharmacist 3)

Meanwhile, nurses expressed a preference for pharmacists to give the complete medication education for patients, yet recognised real limitations.

Practically, a nurses is the one being always with the patient and they are the one to give more information, what if there is some inquiry-at that time the doctor or the educator or the pharmacist will not be there-of course, they will ask us only so we are the one to give the information. (Focus Group 1, Nurse 5)

\section{DISCUSSION}

A fundamental responsibility of healthcare providers is to give timely and appropriate information to patients. We found commonalities among the nurses and pharmacists interviewed in the organisation and prioritisation of safety information that they communicated to patients with cancer in this setting. Both health professional groups informed patients of the common adverse effects to anticipate, but offered this education with relevant context and reassurance. This demonstrates an effective patient-centred style, taking an individual's thoughts and feelings into account as the information of patients with cancer can be unique, change over the course of care and with acceptance of their diagnosis. ${ }^{23}$

The body of risk communication research has demonstrated that the way adverse effect information is presented affects patient decision-making. In our discussions, no pharmacist or nurse explicitly described giving estimated numeric rates of side effects, although this information may be contained in certain written materials that sometimes accompany counselling patient encounters. In such cases, it has been shown that patients are often unable to correctly interpret their incremental chance of experiencing an unfavourable event compared to placebo, thus perceiving an overestimate of their total risk. ${ }^{24}$ As expected, some nurse and pharmacist participants expressed a reluctance to discuss potential side effects for fear that such information may alarm certain patients and promote non-adherence. While it is true that the disclosure of adverse effect risk has the potential to result in unintended behaviour, it has been demonstrated that people respond favourably when risk information is framed positively $(90 \%$ or 90 out of 100 patients WILL NOT experience the intolerance instead of $10 \%$ of 10 out of 100 patients WILL). ${ }^{25}{ }^{26}$ Other research has shown beneficial effects on patient's perceived risk of adverse effects when use of personal terms, such as 'your symptoms', as opposed to impersonal 'the symptoms' are employed and when interpretations are supported by visual (graphs, pictograms). ${ }^{24}$ Pharmacists and nurses in our focus groups were generally not dissuaded from sharing risk information, but recognised that the possibility of subsequent patient non-adherence could be mitigated by choosing side effects patients would most likely immediately experience and coupling this information with education related to their management.

The level of cohesion in safety education prioritised by these two health disciplines may be surprising, given the diversity of the expatriate workforce on which Qatar relies for the delivery of health services. Different cultures appoint different weights to information on patients with cancer regarding diagnosis, prognosis and treatment. ${ }^{14}$ We did not formally collect ethnic background in our study demographics, but the nursing population in Qatar is predominantly Southeast Asian, where in many of these countries the role of the oncology nurse is established as a caregiver delivering treatment and education, but they also face challenges with cultural norms. ${ }^{27}$ As expected, pharmacists and nurses both described efforts, and often creativity, in working around medication counselling of patients unaware of their cancer diagnosis. This convention has been described elsewhere where the culture of non-disclosure predominates. For example, in many Asian, European and Latin countries, patients with cancer are not often fully informed of their condition. ${ }^{14}{ }^{28-30}$ However, this approach is often found to be at odds with the wishes of such patients when they are surveyed. ${ }^{12} 31$ Work exploring preferences among Middle Eastern Arab populations is only just emerging. In a study among Saudi Arabian nationals with symptoms suspicious for malignancy who were referred for cancer diagnosis, all rejected the withholding of information regarding diagnosis and the majority $(99 \%)$ wanted to know the benefits and adverse effects of therapy. Level of education did not appear to be a factor influencing participants' desire for transparency as half of those participating were characterised by investigators as illiterate. ${ }^{15}$

The pharmacists and nurses we interviewed conceivably use professional judgements drawn from a body of experience in cancer care, which ranged from 8 months to 20 years; however, we know that these assessments can be incompatible with patient information priorities and information preferences cannot be simply distilled through a uniform cultural characteristic. At least one instrument has been developed to aid efforts to systematically assess level of individual patient education and information needs, but it is not known how widely it has been adopted in clinical practice. ${ }^{32}$

The health professionals interviewed all viewed themselves as having important responsibilities in communicating safety education, but some responses imply that pharmacists may minimise the role of nurses. Understanding each professional's role can minimise gaps in and ensure continuity of care, especially when there exists an overlap in scopes of practice. ${ }^{33}$ The current professional conditions are such that pharmacists in this and some other Middle Eastern countries are reshaping traditional care identities and may be 
concentrating on establishing their own expanded autonomous roles. ${ }^{34}$ Inherent in their training, pharmacists traditionally have the most structured approach to communicating information pertaining to drugs. Also, at this care site, only a small number of nurse educators have defined patient teaching roles and the pharmacists would not necessarily witness patient follow-up with bedside nurses. This ongoing informal reinforcement by nurses is invaluable for continuity of care, especially as patients often forget information over time. ${ }^{35}$ Both groups agreed that the physician's role in educating patients is incomplete and attributed this to several factors: low patient retention of information from that first encounter with the physician; physician emphasis on explanation of diagnosis and gaining consent to care; physician discretion that the patient does not need or wish to have the information; and finally lack of physician time resulting in (communicated and uncommunicated) delegation to other care providers.

The interpretation of our results is subject to the limitations of all small-scale qualitative work; generalising findings rests on theoretical, rather than, statistical inferences. The six focus group discussions involved proportionately more members of overall pharmacy personnel (total staff 32) compared to nursing (total staff 263), so it is possible that we did not exhaust all possible themes. While participant numbers within our pharmacist groups were smaller than traditionally recommended for focus groups $(<8$ individuals), smaller or 'mini-focus groups' may be advantageous in ensuring that all members have an opportunity to contribute and offer investigators greater insight. ${ }^{36}$

Our participants are from a single geographic area and cannot be assumed to represent similar populations regionally or otherwise. However, we feel that the concepts arising from our work contribute to the dearth of literature related to communication of medication safety and tolerability information to patients with cancer by multidisciplinary team members. Participants offered recommendations to augment such education for patients in this setting, many that are consistent with patient-centred care concepts and recommendations made in the National Cancer Strategy. ${ }^{37}$ Development of a more formal structure should first further consider the views of both physicians and patients in this setting and not obviate the ability for informal education by providers throughout the patient care process.

\section{CONCLUSION}

Nurses and pharmacists in this Middle East healthcare setting assume responsibilities in treatment risk communication with patients. They are not reluctant to discuss side effects, but both disciplines draw on similar professional judgements in prioritising information and framing the education to be most appropriate for the specific patient. We found that they did not always recognise each other's informal educational encounters and there are opportunities to explore increased collaboration in this regard to enhance the patient care experience.

Contributors The project was designed and developed by KW and SMASA-Y. Data were collected by AB, EJ, MA-O, KW and AJN and analysis was led by $A B, E J, M A-0$ and KW. All authors contributed to the drafting and revision of the manuscript and approved the final version. $A B$, EJ and $M A-0$ were undergraduate students training in qualitative research under the supervision of the faculty member, KW.

Funding This publication was made possible by the Undergraduate Research Experience Program award (UREP 14-001-3-001) from the Qatar National Research Fund (a member of Qatar Foundation). The statements made here are solely the responsibility of the authors.

Competing interests None.

Ethics approval Qatar University and Hamad Medical Corporation.

Provenance and peer review Not commissioned; externally peer reviewed.

Data sharing statement No additional data are available.

Open Access This is an Open Access article distributed in accordance with the Creative Commons Attribution Non Commercial (CC BY-NC 4.0) license, which permits others to distribute, remix, adapt, build upon this work noncommercially, and license their derivative works on different terms, provided the original work is properly cited and the use is non-commercial. See: http:// creativecommons.org/licenses/by-nc/4.0/

\section{REFERENCES}

1. Belvedere $\mathrm{O}$, Minisini $\mathrm{A}$, Ramello $\mathrm{M}$, et al. Information given to cancer patients on diagnosis, prognosis and treatment: the clinical oncologist's perspective. Eur J Cancer 2004;40:1850-4.

2. Read H, Ladds $\mathrm{S}$, Rhodes $\mathrm{B}$, et al. The impact of a supplementary medication review and counselling service within the oncology outpatient setting. Br J Cancer 2007;96:744-51.

3. Conron M, Phuah S, Steinfort D, et al. Analysis of multidisciplinary lung cancer practice. Intern Med J 2007;37:18-25.

4. Vinod SK, Sighorn MA, Delaney GP. Do multidisciplinary meetings follow guideline-based care? J Oncol Practice 2010;6:276-81.

5. Wheless SA, McKinney KA, Zanation AM. A prospective study of the clinical impact of a multidisciplinary head and neck tumour board. Otolaryngol Head Neck Surg 2010;143:650-4.

6. Husson O, Mols F, van de Poll-Franse LV. The relation between information provision and healh-related quality of life, anxiety, and depression among cancer survivors: a systematic review. Ann Oncol 2011;22:761-72.

7. Jones RB, Pearson J, Cawsey AJ, et al. Effect of different forms of information produced for cancer patients on their use of the information, social support, and anxiety: randomised trial. BMJ 2006;332:942-8.

8. Mallinger JB, Griggs JJ, Shields CG. Patient-centered care and breast cancer survivors'satisfaction with information. Patient Educ Couns 2005;57:342-9.

9. Harris KA. The informational needs of patients with cancer and their families. Cancer Pract 1998;6:39-46.

10. Kent EE, Arora NK, Rowland JH, et al. Health information needs and health-related quality of life in a diverse population of long-term cancer survivors. Patient Edu Couns 2012;89:345-52.

11. Koinberg I, Langius-Eklof A, Holmberg $L$, et al. The usefulness of a multidisciplinary educational programme after breast cancer surgery: a prospective and comparative study. Eur J Oncol Nurs 2006;10:273-82.

12. Piredda M, Rocci L, Gualandi R, et al. Survey on learning needs and preferred sources of information to meet these needs in Italian oncology patients receiving chemotherapy. Eur J Oncol Nurs 2008;12:120-6.

13. Guleser GN, Tasci S, Kaplan B. The experience of symptoms and information needs of cancer patients undergoing radiotherapy. $J$ Cancer Educ 2012;27:46-53.

14. Dein S. Explanatory models of and attitudes towards cancer in different cultures. Lancet Oncol 2004;5:119-24.

15. Al Amri AM. Cancer patients' desire for information: a study in a teaching hospital in Saudi Arabia. Eastern Mediterranean Healh $\mathrm{J}$ 2009;15:19-24. 
16. Brown R, Kerr K, Haoudi A, et al. Tackling cancer burden in the Middle East: Qatar as an example. Lancet Oncol 2012;13:e501-e8.

17. Silbermann M, Pitsillides B, Al-Alfi N, et al. Multidisciplinary care team for cancer patients and its implementation in several Middle Eastern countries. Ann Onc 2013;23(Suppl 7):vii41-47.

18. Zheng R, Johnson J, Qang Q. A need for cancer patient education from the perspective of Chinese patients and nurses: a comparison study. Support Care Cancer 2014;22:2457-64.

19. Guest G, MacQueen KM, Namey EE. Applied thematic analysis. Los Angeles, USA: Sage Publications Inc, 2012.

20. Krueger RA, Casey MA, eds. Focus groups. A practical guide for applied research. 4th edn. Los Angeles, USA: Sage Publications Inc, 2009.

21. Braun V, Clarke V. Using thematic analysis in psychology. Qual Res Psychol 2006;3:77-101.

22. Bazeley P. Qualitative data analysis. Practical strategies. London, UK: Sage Publications Ltd, 2013.

23. Fervers B, Leichtnam-Dugarin L, Carretier J, et al. The SOR SAVOIR PATIENT project-an evidence-based patient information and education project. Br J Cancer 2003;89(Suppl 1):S111-6.

24. Zikmund-Fisher BJ, Fagerlin A, Roberts TR, et al. Alternate methods of framing information about medication side effects: incremental risk versus total risk of occurrence. J Health Commun 2008;13:107-24.

25. Berry DC. Informing people about the risks and benefits of medicines: implications for the safe and effective use of medicinal products. Curr Drug Saf 2006;1:121-6.

26. Taylor J. Framing the risk of an OTC medication side effect. Can Pharm J 2011;144:34-9.
27. Quinn A. Expanding the role of the oncology nurse. Biomed Imaging Interv J 2008;4:e34

28. Faria SL, Souhami L. Communication with the cancer patient: information and truth in Brazil. Ann NY Acad Sci 1997:809:163-71.

29. Uchitome $Y$, Yamawaki S. Truth-telling practice in cancer care in Japan. Ann NY Acad Sci 1997;809:7-16.

30. [No authors listed]. Awareness of disease among Italian cancer patients: is there a need for further improvement in patient information? The Italian Group for the Evaluation of Outcomes in Oncology (IGEO). Ann Oncol 1999;10:1095-100.

31. Rutten LJF, Arora NK, Bakos AD, et al. Information needs and sources of information among cancer patients: a systematic review of research [1980-2003]. Patient Educ Couns 2005;57: 250-61.

32. Arraras JI, Kuljanic-Vlasic K, Bjordal K, et al. EORTC QLQ-INFO26: a questionnaire to assess information given to cancer patients a preliminary analysis in eight countries. Psychooncology 2007;16:249-54.

33. Wilcoxon $\mathrm{H}$, Luxford K, Saunders $\mathrm{C}$, et al. Multidisciplinary cancer care in Australia: a national audit highlights gaps in care and medico-legal risk for clinicians. Asia Pac J Clin Oncol 2011;7:34-40.

34. Kheir N. An Arab pharmacy spring: taking matters in their own hands. Int J Clin Pharm 2013;35:665-7.

35. Ancel G. Information needs of cancer patients: a comparison of nurses' and patients' perceptions. J Cancer Edu 2012;27:631-40.

36. Litamputtong P. Focus group methodology. Principle and practice. Los Angeles, USA: Sage Publications Inc, 2011.

37. National Cancer Strategy. The path to excellence. Doha, Qatar, 2011. 